\title{
Modelling of PID Speed Control Based Collision Avoidance System
}

\author{
L. M. Keong ${ }^{1}$, A. S. Jamaludin ${ }^{1 *}$, A. N. S. Z. Abidin ${ }^{1,2}$, M. N. N. Razali1 ${ }^{1}$ M. R. M. Yasin ${ }^{1}$ \\ ${ }^{1}$ Faculty of Manufacturing and Mechatronic Engineering Technology, Universiti Malaysia Pahang, 26600 Pahang, Malaysia. \\ ${ }^{2}$ Malaysian Institute of Road Safety Research (MIROS), Lot 125-135, Jalan TKS 1, Taman Kajang Sentral, 43000 Kajang, Selangor, Malaysia
}

ABSTRACT - To date, motor vehicle collisions in Malaysia is still considerably high particularly during festive seasons. These collisions may have been contributed by various factors viz road users, the vehicles or due to the road design. To reduce the possibilities of vehicle collisions, vehicle manufacturers have incorporate various types of active safety features as part of their vehicle specifications. However, cost seems to be a continous matter of argument among the industries, authorities and consumers. To ensure the sustanability and practicality of such features, it is necessary to develop a low cost yet high effectiveness collision avoidance system that is affordable in every vehicles. Therefore, this research focuses in designing and modelling a low cost collision avoidance system that will decelerate vehicles when approaching on-road obstacles. The effectiveness of this collision avoidance system is evaluated based on its effectiveness in slowing down vehicles when the system detects obstacles in front, similar to the Autonomous Emergency Braking (AEB) features in vehicles which has now becoming more and more common for new models. In this research, the collision avoidance system will be tested on a model car that is built with robot car chassis. The Arduino Nano acts as the controller for the system while ultrasonic sensors and encoder sensors are used to detect the distance of obstacles and speed of the wheels respectively. The Arduino board is programmed with PID control algorithm to allow the model car to decelerate when approaching obstacle and come to complete stop before colliding with the obstacles. According to the result obtained, the most suitable PID constants for this collision avoidance system is $\mathrm{Kp}=0.7, \mathrm{Ki}=0.7$ and $\mathrm{Kd}=0.07$. The collision avoidance system is sensitive to sudden obstacles as it can stop the model car within 0.3 second from obstacle detection. To conclude, this system can effectively avoid the model car from crashing into the front obstacle thus may be beneficial as reference for low cost collision avoidance technologies for commercial purposes in motor vehicle technologies.

\section{ARTICLE HISTORY}

Revised: $30^{\text {th }}$ September 2020

Accepted: $30^{\text {th }}$ September 2020

\section{KEYWORDS}

Road safety

Smart Car

PID Controller

Collision Avoidance

Smart Braking System

\section{INTRODUCTION}

The journey of autonomous vehicles development began as early as 1926 with the introduction of the world's first radio controlled car. Ever since, the development works continued and autonomous cars were already travelling on public highways by the year 1959. Meanwhile, the first introduction of vision based system in vehicles happened in the year 1980 which was regarded as the greatest achievement in field of autonomous vehicles [1]. Additionally, many architectures for autonomous vehicle features were introduced since then with the new enabling technologies, platforms and programs. Nowadays, the architecture of autonomous vehicles is developed from radio controlled car to connected cars through vehicular communication and cloud computing. The initial objectives of the development of autonomous cars were to enable safe autonomous driving, while less human interaction thus reducing human errors. The safety of the drivers and passengers of the vehicle is always the main concern for the development of the autonomous vehicles.

In order to reduce the possibility of road collisions, many safety features developed by vehicle manufatcurers for the sake of improving vehicle control and manuevering during emergency situations. These features are technically known as active safety systems. Among the examples of the active safety features being introduced so far are collision avoidance system, adaptive steering control, autonomous advanced braking system, antilock braking system, blindspot detection system and many others. These system are designed to avoid the collisions either with another vehicle or an obstacle through more controllable manuever by the driver or by decelarating the vehicle to a stop to mitigate the risk of collisions. Together with the architecture of connected cars or cloud computing systems for the autonomous vehicles, vehicle braking system also plays a major role [3].

To date, vehicle manufacturers have come out with a number of braking systems such as the integrated electronic hydraulic brake system, electric booster brake system, initiative braking system and others. However, the systems are not automatically applied thus requiring driver input. On the other hand, vehicle safety engineers have also introduced several smart braking systems, for example speed control smart automatic braking system and servo motor torque controller for autonomous vehicle and name a few [4]. These systems posses similarity in features, in which the usage of PID controller 
in the systems. PID controller is the proportional-integral-derivative controller which is a mechanism device to control the loop that involves feedback into the system. An accurate and responsive correction according to proportional, integral and derivative will be applied to a control function. The PID controllers are able to provide speed control of the motor in order to avoid collisions [5] .

On the downside of all of these features, some of the safety features come with significant cost particularly which involves automated input from the vehicles. These luxury add on are not affordable for all the vehicle segments [5]. On top of that, some of the features also requires costly and high complexity systems for example, stable internet connection in vehicles, numerous numbers of sensors and others. Moreover, some of the information collected need to be transfer to data storage which then the cloud computing service will need to analyse the data collected and send it back to the vehicle for response. All these processes require high upload and download speed equipment and internet services [5].

In the same note, an automatic system is required to ensure the safety of drivers or the passengers of a vehicle, as the alert system may not help if the driver were in panic or emergency situations [7]. Moreover, the aforemention electrical braking systems developed are mostly not automated, thus requiring human interaction to manually press the brake pedal to apply braking force in order to stop the vehicle [8]. Thus, the needs to develop a low cost high effectiveness collision avoidance system that are manageable yet affordable, such as PID controller based collision avoidance system is needed. With such system developed, it is hoped that it will help in reducing the probability od vehicle collisions and the usage of the system can be broaden to a wider scope in vehicle segments.

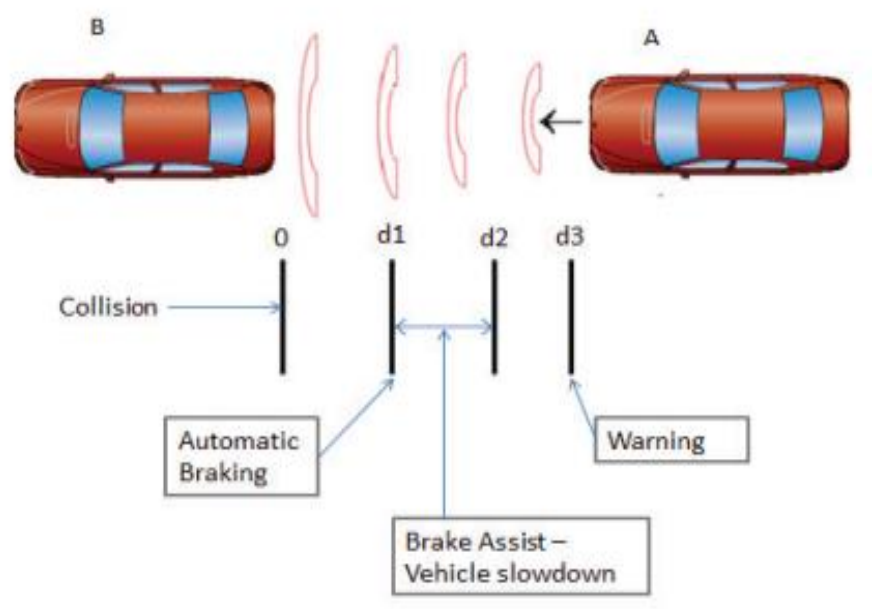

Figure 1: Graphical Illustration of Working Principle [5]

In this study, of collision avoidance system involving PID algorithm control to provide accurate speed control and avoid collision is modelled. A model car will be assembled and programmed with PID control based Arduino program. The speed of the model car will be controlled according to the frontal obstacle's distance. The collision avoidance system will be developed using an Arduino Nano board, ESP8266 Wi-Fi module, modelling car chassis, motor driver, ultrasonic sensor, encoder sensors, LCD display and other components. This collision avoidance system which involved PID controller is expected to be able to decelerate and stop the vehicle thus avoiding frontal collisions with obstacles or rear end collisions with other vehicles.

\section{METHODOLOGY}

This research focuses in developing and modelling the collision avoidance system that is able to decelerate vehicle when approaching obstacles in order to avoid collisions. The collision avoidance system will be tested using the model car as shown in Figure 2 and 3. The PID control algorithm will be programmed into Arduino Nano to control the speed of DC motor according to the situation provided. A model of vehicle will be built using the Arduino Robot car chassis, and the model car will be controlled through Bluetooth connection. The Bluetooth control application will be used to control the model car during experiment to test the functionality and effectiveness of the system. The distance of obstacle and the real time speed of the model car will be displayed using LCD monitor, so the speed changes could be monitored. The distance and speed data of the model car will be saved in the online database for data analysis purpose. 


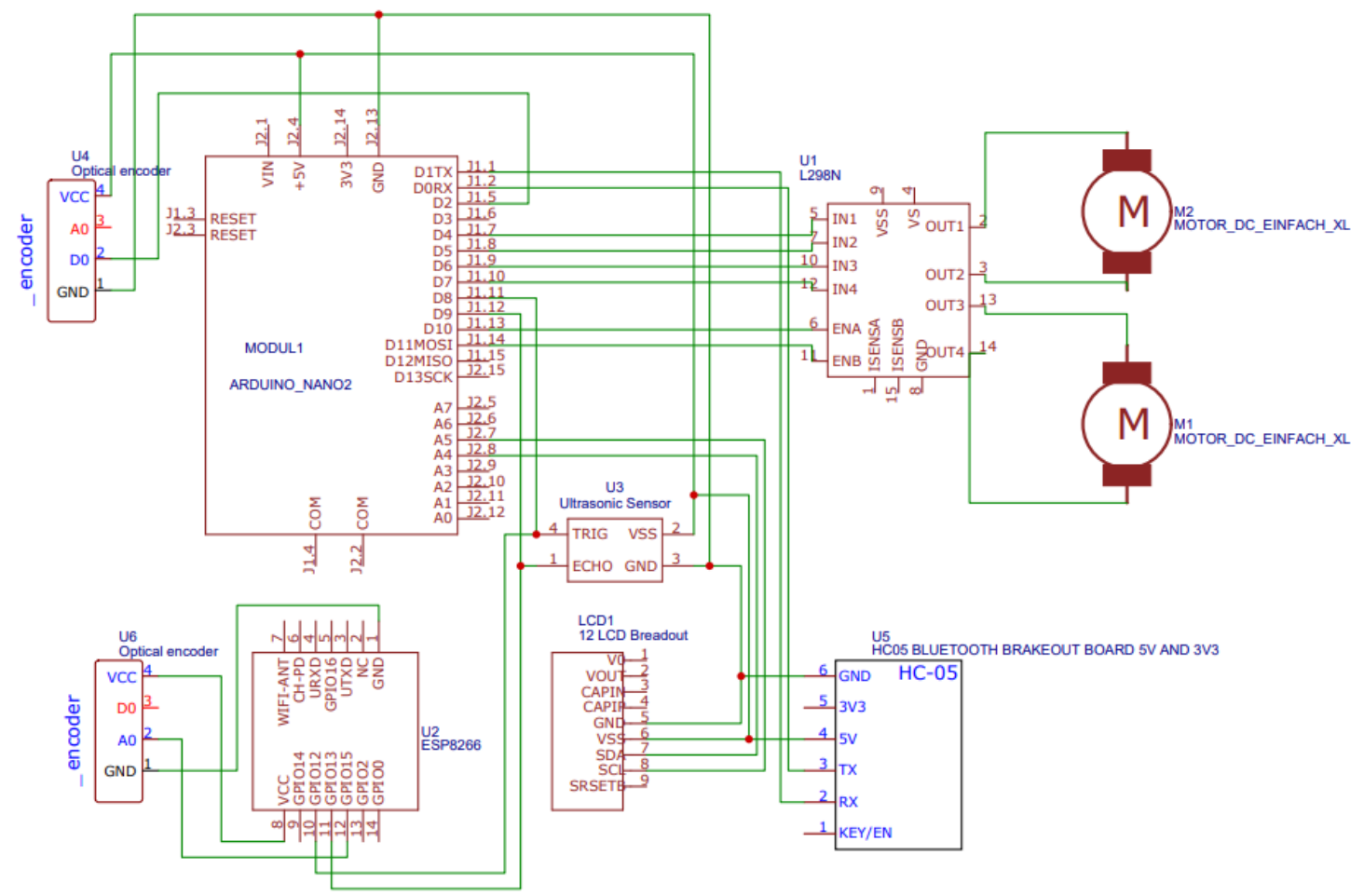

Figure 2: Model car installed with PID control collision avoidance system

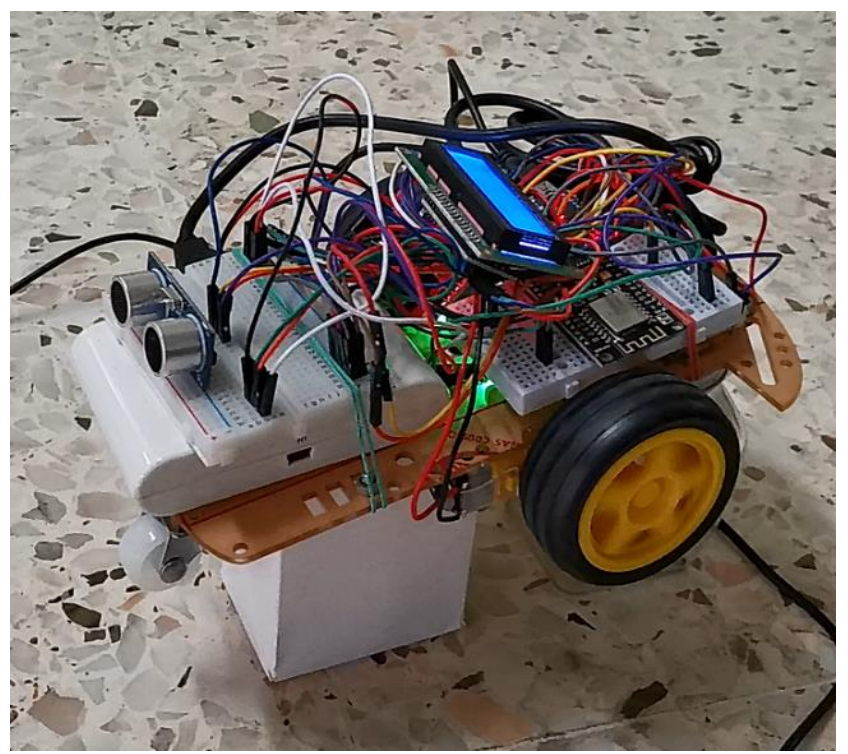

Figure3 : Model car installed with PID control collision avoidance system

The effectiveness of the collision avoidance system will be investigated through several experiments. Firstly, the system will be tested under different sets of PID constant values as shown in Table 1 while the most suitable PID constant values will be determined through trial and error experiments. Next, the model car will be programmed with collision avoidance system with and without PID controller. Their performance of speed reduction when approaching the obstacles will be compared and analyzed. Thirdly, the PID control based collision avoidance system will be tested under different initial speed so that its functionality under different initial speed of 100 RPM, 150 RPM, 200RPM and 250 RPM are observable. Lastly, an experiment will be carried out to test on the effectiveness of the collision avoidance system to react to the sudden obstacles under different speed. All the process flowchart for software and hardware are shown by Figure 2 and 3 respectively. 
Table 1: PID constants value set for experiment

\begin{tabular}{cccc}
\hline Set & $\mathrm{Kp}$ & $\mathrm{Ki}$ & $\mathrm{Kd}$ \\
\hline 1 & 1 & 0 & 0 \\
\hline 2 & 1 & 1 & 0 \\
\hline 3 & 1 & 1 & 1 \\
\hline 4 & 0.7 & 0.7 & 0.07 \\
\hline
\end{tabular}

\section{RESULT AND DISCUSSIONS}

\section{Study on the effects of different of PID constants to the speed control}

The speed responses against distance of the model car installed with PID control collision avoidance system is displayed in Figure 4. For the first set of PID constants which are Speed Response Constant Kp=1, Total Distance Respone Constant $\mathrm{Ki}=0$ and Acceleration Response Constant $\mathrm{Kd}=0$, the speed response is represented by the green graph. The graph is not plotted out because the speed value is zero. According to PID equation shown in Equation (1), the integral term can eliminate the steady state error over time, the car is not moving is due to zero of integral term.

$$
u(t)=K_{p} e(t)+K_{i} \int_{0}^{t} e\left(t^{\prime}\right) d t^{\prime}+K_{d} \frac{d e(t)}{d t}
$$

For the second set of PID constants which are $\mathrm{Kp}=1, \mathrm{Ki}=1$ and $\mathrm{Kd}=0$, the speed response is represented by the blue graph. The graph shows that the car is moving with fluctuating speed and finally crashing to the obstacles. The derivative term can help the system to respond to the changes of speed. The derivative term for this case is zero and cause it crashing to the obstacle. Next, the yellow graph represents the speed response with the set 3 of PID constants, $\mathrm{Kp}=1, \mathrm{Ki}=1$ and $\mathrm{Kd}=1$. The car is moving with a highly fluctuating speed. The unstablity of the system is caused by the Kd value is too high for the system. The fourth set of PID constants, $\mathrm{Kp}=0.7, \mathrm{Ki}=0.7$ and $\mathrm{Kd}=0.07$ is the most suitable value for this collision avoidance system. The speed of response is represented by the red graph, it shows that the car is slowing down gradually when approaching the obstacle and stop before colliding with the obstacles.

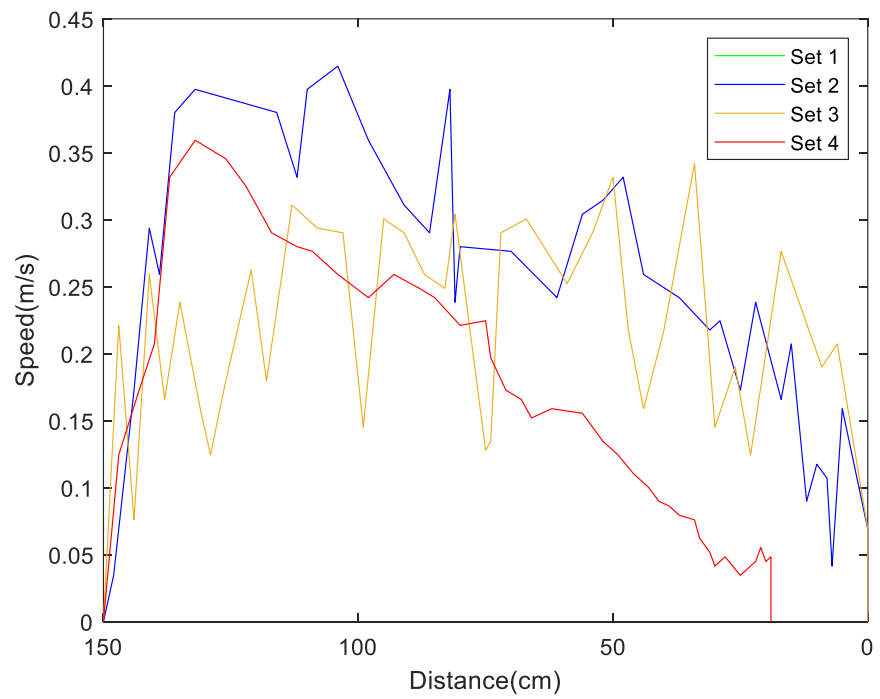

Figure 3: Graph of Speed against Distance on different set of PID constants

\section{Comparison of collision avoidance system with and without PID controller.}

The speed response of model car is programmed with collision avoidance system with and without PID controller are displayed in the Figure 5. The speed reducing trend can be observed from both of the graphs. However, the model car without PID controller algorithm reduce the speed and stop abruptly, while the car slow down gradually when approaching obstacle with PID controller algorithm. In additional, the modelling car stop abruptly at a $40 \mathrm{~cm}$ obstacle distance. It happens mainly due to the insufficient current supplied to move the DC motors, the insufficient current is caused by the low PWM values set when the modelling car gets closer to the obstacle. This issue can be solved by having the PID controller algorithm as the integral term of the PID controller can provide an accumulated offset to supply sufficient 
current to move the model car even with the low PWM values. From the comparison of both graphs, the effectiveness and benefits of having the speed controller algorithm in the model car are clearly proved.

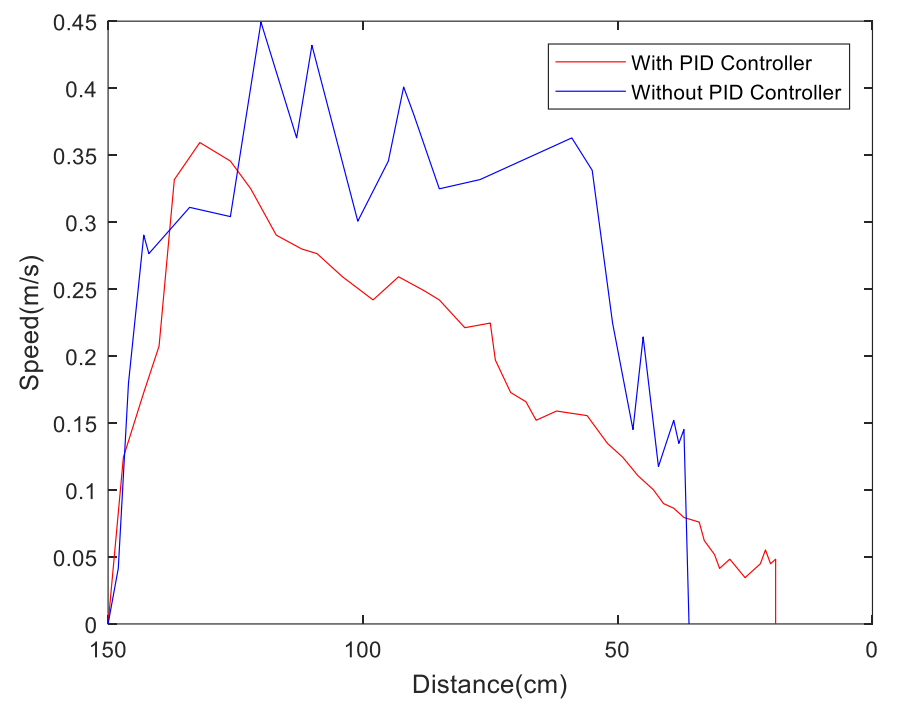

Figure 4: Graph of Speed against Distance for modelling car with and without PID controller

\section{Study on effectiveness of collision avoidance system on various initial speed}

The relationship between speed and distance of the model car with different initial speed are shown in Figure 6. From the graph, it is observed that the speed of the model car is reduced gradually when approaching obstacles even with different initial speed. However, steady state error is pbserved in the graph whereby the value seems to be fluctuating at the end of the graph. The low steady state error can be considered acceptable as the main objective of this collision avoidance system is to stop the car before colliding with the obstacle. From the graph, it is observed that the model car is able to stop before colliding with obstacle in various speeds and good speed reduction characteristics can be observed. If the initial speed increases, the model car also decelerates greatly as it approaches closer to the obstacle. The experiment had proved prove that the PID control collision avoidance system are able to reduce the speed gradually or sharply to prevent collisions with the front obstacle with different initial speed of the modelling car.

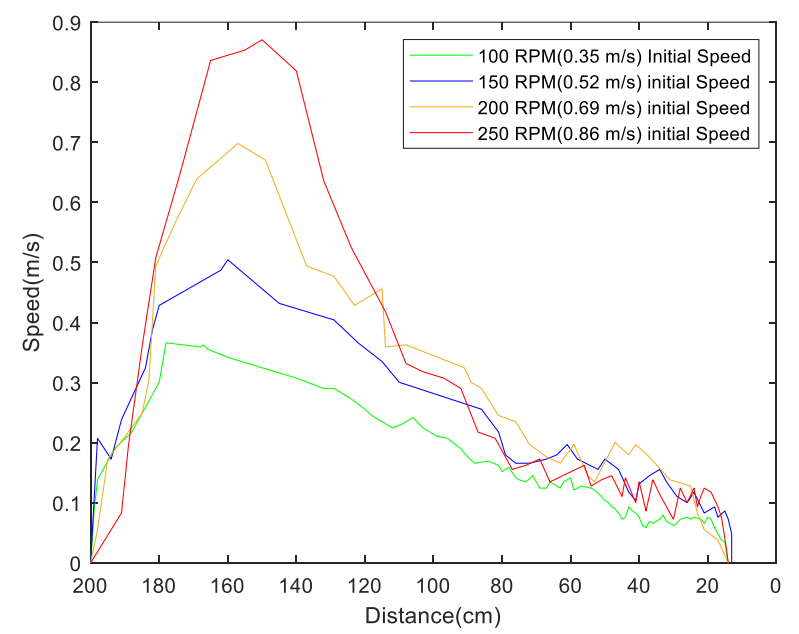

Figure 5: Graph of Speed against Distance with different initial speed. 
Study on effectiveness of sudden braking on different initial speed.

Table 2: Time to stop the wheel when obstacle detected under different initial speed

\begin{tabular}{cc}
\hline Initial speed (RPM) & Time to stop the wheel when obstacle detected (second) \\
\hline 100 & 0.4 \\
\hline 150 & 0.3 \\
\hline 200 & 0.3 \\
\hline 250 & 0.3 \\
\hline
\end{tabular}

Table 2 shows the experiment result of the modelling car after being tested with the sudden braking experiment. The experiment is done to observe reaction time of the PID controller to stop the wheel of the modelling car. With 100 RPM initial speed, it requires only 0.4 second to stop the wheel of the modelling car once the obstacles is detected. Next, the system can reduce the speed to zero with 0.3 second only for the experiment of initial speed of 150 RPM, 200 RPM and 250 RPM. The experiments show that the system is sensitive to the changes of the obstacle distance and able to stop the vehicle if sudden obstacle appear in front the modelling car.

\section{Discussion the Collision Avoidance System implemented on Real vehicles}

The PID speed control collision avoidance system is practical to be installed on real vehicles as experiments proved that the collision avoidance system can delecerate vehicles to a stop thus avoiding collisions. If it is implemented on the vehicles with combustion engines, the controller can be used to control the braking system of the vehicles. On the other hand, for electric vehicles, the controller will control the speed of DC motor similar to this research. Since braking system is still implemented on the electric vehicles, it provides opportunity for the controller to control both the braking system and DC motor to achieve the best speed control for the electric vehicles. However, the PID constants are required to be further tested by considering the vehicle parameter if is to be installed on real vehicles.

\section{CONCLUSION}

From the experiments result, it was proved that the model car that was installed with PID control collision avoidance system is able to be decelerated when approaching obstacles and stopped before colliding with the obstacles. After several trial and error testings, the most suitable PID constants for this model car collision avoidance system is $\mathrm{Kp}=0.7, \mathrm{Ki}=$ 0.7 and $\mathrm{Kd}=0.07$. These values provide the optimum performance of the system with safety and comfort as the outcome. Moreover, the model car is programmed with and without PID control algorithm and the comparison of the performance of speed reduction was conducted. The graphical result shows that the model car with PID control algorithm possessed better speed reduction behaviour as it reduces speed slowly while the model car without PID control algorithm reduces speed abruptly. Further to that, the model car with PID control collision avoidance system is also tested on different initial speeds. The experiment result showed that the collision avoidance system was capable to reduce the speed of the model car and avoid obstacle collision under different initial speeds. Subsequently, the collision avoidance system is tested to measure its effectiveness in reacting to sudden obstacles. The results have shown that the system could stop the wheel of the model car by nearly 0.3 seconds once the sudden obstacle was detected close to the ultrasonic sensors. Thus, to conclude the system is practical and effective to serve its intent purpose thus may be considered to be installed on real passenger vehicles with minor variable settings required to suit the vehicle.

\section{ACKNOWLEDGEMENT}

The authors are grateful to the Ministry of Higher Education (MOHE) for financially supported this study through RACER - Fundamental Research Grant Scheme (RACER/1/2019/TK05/UMP//1) - RDU192621 and Universiti Malaysia Pahang for providing the financial assistance through Postgraduate Research Scheme PGRS1903177. 


\section{REFERENCES}

[1] Bimbraw K (2015) Autonomous Cars : Past, Present and Future. 12th Int Conf Informatics Control Autom Robot 01:191198

[2] Hamid UZA, Zakuan FRA, Zulkepli KA, Azmi MZ, Zamzuri H, Rahman MAA, Zakaria MA (2018) Autonomous emergency braking system with potential field risk assessment for frontal collision mitigation. Proc - 2017 IEEE Conf Syst Process Control ICSPC 2017 2018-Janua:71-76 . https://doi.org/10.1109/SPC.2017.8313024

[3] Kong L, Khan MK, Wu F, Chen G, Zeng P (2017) Millimeter-wave wireless communications for IoT-cloud supported autonomous vehicles: Overview, design, and challenges. IEEE Commun Mag 55:62-68 . https://doi.org/10.1109/MCOM.2017.1600422CM

[4] More HR, Digrase AA, Wayse AV (2017) Linear PID control technique for single wheel ABS (anti-lock braking system) of motorcycle. 2017 2nd Int Conf Converg Technol I2CT 2017 2017-Janua:277-281 https://doi.org/10.1109/I2CT.2017.8226135

[5] Rezwan MS, Islam MA, Islam MM, Hasan MR (2018) Vehicle Breaking Support System. 2018 3rd Int Conf Converg Technol I2CT 2018 1-4

[6] Mallikarjuna, G. C. P., Hajare, R., Mala, C. S., Rakshith, K. R., Nadig, A. R., \& Prtathana, P. (2018). Design and implementation of real time wireless system for vehicle safety and vehicle to vehicle communication. International Conference on Electrical, Electronics, Communication Computer Technologies and Optimization Techniques, ICEECCOT 2017, 2018-Janua, 354-358

[7] Pi, D., Cheng, Q., Xie, B., Wang, H., \& Wang, X. (2019). A Novel Pneumatic Brake Pressure Control Algorithm for Regenerative Braking System of Electric Commercial Trucks. IEEE Access, 7, 83372-83383

[8] Y. Qian, X. Yang, L. Xiao, and Y. Zhu, "Optimization of Braking Strategy for Automatic Emergency Braking System in Vehicle-Pedestrian Accidents," SAE International Journal of Passenger Cars - Electronic and Electrical Systems, vol. 12, no. 2, 2020. 